\title{
High Resolution Computerized Tomography of the Chest and Pulmonary Function Testing in Evaluating the Effect of Tobramycin Solution for Inhalation in Cystic Fibrosis Patients
}

\begin{abstract}
Samya Z. Nasr, MD, ${ }^{1 \star}$ Diane Gordon, RN, MS, MPH, ${ }^{4}$ Ermelinda Sakmar, MA, RN, ${ }^{1} \mathrm{Xin} \mathrm{Yu}, \mathrm{PhD},{ }^{5}$ Emmanuel Christodoulou, $\mathrm{PhD},{ }^{3}$ Boris P. Eckhardt, $\mathrm{MD}^{2}{ }^{2}$ and Peter J. Strouse, $\mathrm{MD}^{2}$

Summary. To evaluate the sensitivity of high-resolution computerized tomography (HRCT) of the chest compared to spirometry measures in evaluating the effects of tobramycin solution for inhalation (TSI) in cystic fibrosis (CF) patients. Thirty-two subjects $\geq 6$ years old with mild to moderate CF lung disease were enrolled in a randomized, double-blind, placebo-controlled pilot study. Duration was 28 days; 31 subjects completed the study.HRCTscores decreased $4.06 \pm 3.20$ (mean $\pm \mathrm{SD}$ ) for TSI and decreased $0.17 \pm 1.78$ for placebo subjects $(P=0.13)$. Mean forced expiratory flow during middle half of forced vital capacity $\left(\mathrm{FEF}_{25 \%-75 \%}\right)$ predicted increased $6.08 \pm 4.86$ for TSI and decreased $0.60 \pm 2.34$ for placebo $(P=0.23)$. Percentage forced expiratory volume in $1 \mathrm{~s}\left(\mathrm{FEV}_{1}\right)$ predicted increased slightly for both $\mathrm{TSI}$ and placebo $(1.29 \pm 3.33$ for TSI and $1.17 \pm 1.4$ for placebo $)(P=0.97)$. Two of eight HRCT component scores (atelectasis and inhomogeneity) were observed to be highly discordant with observed HRCT global total score and other HRCT component scores. A modified total score was calculated by dropping them from the global total score. The modified HRCT total scores decreased $6.68 \pm 3.09$ for TSI subjects and increased $0.02 \pm 2.0$ for the placebo subjects $(P=0.07)$. Sample sizes were calculated to show statistical significance by differences in modified total HRCTscores, global total HRCT scores, $\mathrm{FEF}_{25 \%-75 \%}$ predicted or $\mathrm{FEV}_{1} \%$ predicted. A total of $60,100,200$, and over 800 patients would be necessary respectively.HRCT can be a useful measure of change in CF pulmonary disease, requiring a smaller sample size than that required to show treatment effect by pulmonary function testing (PFT) alone. Pediatr Pulmonol. 2006; 41:1129-1137.

(c) 2006 Wiley-Liss, Inc.
\end{abstract}

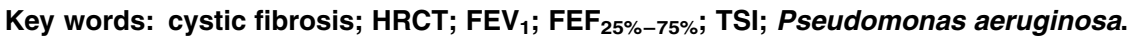

\section{INTRODUCTION}

Progressive destruction of the lungs leads to increased morbidity and mortality in patients with cystic fibrosis (CF). Inflammation and chronic infection of the small airways lead to pulmonary dysfunction. ${ }^{1}$ High resolution

\footnotetext{
${ }^{1}$ Department of Pediatrics, Division of Pediatric Pulmonology, University of Michigan Health System, Ann Arbor, Michigan.

${ }^{2}$ Department of Radiology, Section of Pediatric Radiology, University of Michigan Health System, Ann Arbor, Michigan. ${ }^{3}$ Section of Physics/Engineering, University of Michigan Health System,
Ann Arbor, Michigan.

${ }^{4}$ Chiron Corporation. Currently at Department of Biobehavioral Nursing and Health Systems, School of Nursing, University of Washington, Seattle, Washington.
}

${ }^{5}$ Chiron Corporation, Seattle, Washington.

(C) 2006 Wiley-Liss, Inc. computed tomography of the chest (HRCT) can illustrate the airway involvement characteristic of this disease, especially early changes, and therefore has the potential to be a useful outcome measure in $\mathrm{CF}^{2}$ It is noninvasive, does not require contrast material, and with limited number of cuts, the overall radiation dose is low. ${ }^{3}$ HRCT appears to

Presented in part at the European Respiratory Society Annual Congress, Glasgow, Scotland from September 4 to 8, 2004, and at North American Cystic Fibrosis Conference, St. Louis, MO from October 14 to 18, 2004.

Grant sponsor: Chiron Corp.

*Correspondence to: Samya Z. Nasr, MD, Associate Professor of Pediatrics, 1500 E. Medical Center Drive, Ann Arbor, MI 48109-0212. E-mail: snasr@umich.edu

Received 18 October 2005; Revised 7 March 2006; Accepted 7 March 2006.

DOI 10.1002/ppul.20447

Published online in Wiley InterScience

(www.interscience.wiley.com). 
be a more sensitive measure than CXR in evaluating CF lung disease, especially in patients with mild lung disease. $^{4}$

A recent study demonstrated positive correlation between five different HRCT scoring systems and pulmonary function testing (PFTs), especially forced expiratory volume in $1 \mathrm{~s}\left(\mathrm{FEV}_{1}\right)$, forced expiratory flow during middle half of forced vital capacity $\left(\mathrm{FEF}_{25 \%-75 \%}\right)$, $\mathrm{FEV}_{1} / \mathrm{FVC}$ ratio, and TLC values. ${ }^{5}$ Correlation of the scoring systems with each other and with PFT parameters was very good. ${ }^{5}$ Currently, PFTs are the most accepted proven outcome measure for the evaluation of $\mathrm{CF}$ lung disease. However, because of the slow decline in PFTs, large patient samples are required in clinical trials to detect significant changes when using these measures. ${ }^{6,7}$

Tobramycin solution for inhalation (TSI) has proven effective in CF patients with chronic Pseudomonas aeruginosa (P. aeruginosa) endobronchial infections. ${ }^{8}$ In CF patients with mild lung disease and chronic $P$. aeruginosa lung infection, TSI leads to a significant reduction in respiratory hospitalizations, concomitant antibiotic use, and a trend towards improvement in percent predicted forced expiratory flow $\left(\mathrm{FEF}_{25 \%-75 \%}\right){ }^{9}$

The aim of this pilot study was to assess the sensitivity of HRCT of the chest compared to PFT in evaluating the effects of TSI in CF patients 6 years of age and older with mostly mild to moderate lung disease.

\section{MATERIALS AND METHODS}

\section{Study Design}

A randomized, double-blind, placebo-controlled pilot study was conducted. The study procedures and measures were reviewed and approved by the University of Michigan Institutional Review Board and consent was obtained from patients and/or parents/guardians prior to enrollment in the study.

\section{Study Population}

Patients with $\mathrm{CF} 6$ years of age and older with $\mathrm{FEV}_{1}$ of $45 \%$ predicted or higher, attending the University of Michigan CF Center, were considered eligible for the

\begin{tabular}{|ll|}
\hline \multicolumn{2}{|l|}{ ABBREVIATIONS } \\
$\mathrm{CF}$ & Cystic fibrosis \\
$\mathrm{FEV}_{1}$ & Forced expiratory volume in $1 \mathrm{~s}$ \\
$\mathrm{FEF}_{25 \%-75 \%}$ & Forced expiratory flow during middle half of forced \\
& vital capacity \\
$\mathrm{HRCT}$ & High resolution chest tomography \\
$\mathrm{HU}$ & Hounsfield unit \\
$\mathrm{PFTs}$ & Pulmonary function tests \\
P. aeruginosa & Pseudomonas aeruginosa \\
TSI & Tobramycin solution for inhalation \\
$\mathrm{CFQ}$ & Cystic fibrosis questionnaire \\
\hline
\end{tabular}

study. Thirty-two patients (12 males and 20 females) with a confirmed diagnosis of CF by sweat chloride test and/or genotype, and $P$. aeruginosa colonization in sputum within 6 months of screening and at enrollment were recruited. Exclusion criteria included known hypersensitivity to aminoglycosides or history of Burkholderia cepacia isolated from respiratory cultures. Patients had to be clinically stable prior to enrollment as defined by last hospitalization for pulmonary exacerbation and intravenous antibiotic use at least 2 months prior to enrollment. Oral antibiotics could not be used for at least 1 month prior to enrollment; TSI could not be used for at least 3 months prior to enrollment. All subjects were pancreatic insufficient.

\section{Study Procedures}

Study duration was 28 days and patients were enrolled between February 2002 and August 2003. Patients were evaluated at baseline and again at the end of the study. Eligible subjects were randomized to receive either $5 \mathrm{ml}$ of TSI (treatment) or $5 \mathrm{ml}$ of placebo twice a day, via inhalation therapy. TSI (Chiron Corporation, Emeryville, CA) is a solution of $300 \mathrm{mg}$ tobramycin and $11.25 \mathrm{mg}$ sodium chloride in sterile water; the placebo was chosen for its similar taste to tobramycin and consisted of $1.25 \mathrm{mg}$ quinine sulfate in normal saline. Standard routine $\mathrm{CF}$ care was provided for both groups as directed by the primary pulmonologist. Study medication and placebo were administered using a Pari LC Plus ${ }^{\mathrm{TM}}$ jet nebulizer and a Pulmo-Aide ${ }^{\mathbb{R}}$ compressor. Both TSI and placebo were supplied by Chiron Corporation. The University of Michigan investigational drug services performed the double blinding for the study.

HRCT and spirometry (Sensormedics, Vmax 6200, Yorba Linda, CA), were obtained at baseline and at the end of the study. Spirometry was obtained according to ATS guidelines. CXR was done at baseline for evaluation of lung disease and as part of routine care. Throat/ sputum cultures were obtained prior to enrollment for confirmation of colonization with $P$. aeruginosa. A quality of life questionnaire (cystic fibrosis questionnaire (CFQ)), validated for use in CF patients, ${ }^{10}$ was administered at baseline and at the end of the study to patients and parents/guardians. Adverse events, hospitalizations, concomitant medication use, and school days/work days missed due to illness, were collected at the end of the study.

HRCT scan was performed using $40 \mathrm{~mA}, 1 \mathrm{~s}$ gantry rotation time, and $120 \mathrm{KVp}$ for each slice through the lung. Utilizing a HiSpeed Advantage ${ }^{\mathbb{R}}$ scanner (General Electric Medical Systems, Milwaukee, WI), images were obtained at $1 \mathrm{~mm}$ thickness. Five equally spaced images in inspiration and in expiration, after an AP inspiratory scout view, were obtained for a total of 
10 images. Patients were placed in the supine position and instructed to take a deep breath and hold it for 710 s. During each breath hold, the five inspiratory sections were obtained. The same technique was repeated after patients were asked to exhale to lowest volume and hold their breath for the expiratory sections. Patients practiced the techniques prior to the testing and were monitored by both the radiology technician and the research coordinator. If techniques were suboptimal, patients were instructed to repeat them. The superiormost image was at the upper margin of the posterior $3 \mathrm{rd}$ rib and the inferior-most image was $1 \mathrm{~cm}$ above the diaphragm. The range of the effective radiation dose was estimated to be $0.03-0.05 \mathrm{mSv}$ and the equivalent radiation dose to the entire lung was estimated to be 0.09 mGy. HRCT images were reviewed on a workstation (Sienet Magicview, version 42, Siemens, New York, NY). Images were initially displayed with a window width of 1,000 Hounsfield Units (HU) and level of $-600 \mathrm{HU}$; however, the readers were permitted to modify the window and level of display of individual images and examinations for optimal viewing.

Scoring of the HRCT scans and CXRs were performed by two pediatric radiologists (PJS and BPE). Both readers were blinded to patient name, number, and study date at the time of reading and scoring. Each CXR (conventional analog) was scored twice by each radiologist using the Brasfield scoring method, ${ }^{11}$ and the scores were averaged across the two readers. A CXR score of 25 points indicates a normal finding, the score decreasing with increasing severity of lung disease. The HRCT score was derived from Brody, A.S. and others. ${ }^{7,12-15}$ Each reader read each HRCT twice in a randomized, blinded fashion. Means of the scores for each reviewer were calculated separately and compared between baseline and end of study. Scores of both readers were also combined (four total readings); a mean of the combined score was calculated and compared between baseline and at end of study. One reader read the HRCT scans for the second time within 1-2 weeks from the first reading. The second reader read the HRCT scans for the second time 1-2 months after the first reading.

Each lung (right \& left) was scored for each of the five imaging levels. Eight imaging component categories were scored at each imaging level for each lung: nodular opacities, linear opacities, hyperinflation (lack of normal decrease in volume on expiratory views), peribronchial thickening (abnormal thickening of bronchial walls), atelectasis (areas of collapsed, non-aerated lung), inhomogeneity (variation in aeration producing variation in lung opacity), bronchiectasis (dilated bronchi usually judged relative to diameter of adjacent pulmonary artery branch), and mucus plugging (opacified bronchus/bronchi)... Hyperinflation and inhomogeneity were scored on expiration because the expiratory images showed this finding better. Some inspiratory images showed inhomogeneity as well. The other six component categories were scored on inspiratory images. Scoring ranged from 0 to 4 with 0 indicating a normal finding and 4 indicating severe disease. The HRCT final score of each patient was calculated based on the severity and extent of the morphologic changes, and represented the average score from the two lungs and two readers. Three summary scores were calculated. (1) A component category score: the average of both reader's scores across the five levels within each category and for both lungs, (2) An imaging level score: The average of both reader's scores across the eight components within each level and for both lungs, (3) total global score: the sum of all eight total HRCT category scores or the sum of all five level scores.

\section{Statistical Analysis}

This is a pilot study. Power calculations of sample size were not done. The number of subjects studied was arbitrarily chosen. Frequencies and percentages were calculated for categorical variables; mean, standard deviation, minimum, and maximum were calculated for continuous variables. The baseline analysis was based on 16 patients in each treatment group. End of study analyses included data from 16 patients in the TSI arm and 15 patients in the placebo arm (excluding the drop-out subject in the placebo arm). SAS version 8 software was utilized for all analyses. A two-sided student's $t$-test for two independent samples was used to assess the treatment effect on the primary efficacy variable at a 0.05 significance level. In addition, non-parametric test (Wilcoxon) was performed. Three summary scores were calculated for the HRCT: a category score, a level score, and a total global score. This scoring system was based on that used previously. ${ }^{7,12}$ Additionally, maximal score was determined for both category and level scores. Coefficient of variation and test of differences in mean scores between and within readers (mean $\pm \mathrm{SD}$ ) were used to measure inter/intra-reader variability in scoring CXR and HRCT images. Inter/intra-rater reliability analyses were carried out to test whether there was a difference between the reliability of the scoring for HRCT and CXR. The subjects in both groups were combined to enlarge the sample size for statistical analyses. HRCT scores from both visits and CXR scores from the first visit were averaged for these analyses. Coefficient of variation and test of differences in mean scores between and within readers (mean \pm SD) were done for the total HRCT score, all HRCT subscores, and the CXR scores.

Correlation statistics $\left(\mathrm{R}^{2}\right)$ were used to measure correlation between specific HRCT component scores and the global total HRCT scores. The coefficient of 
variation was determined according to the following formula:

$$
\begin{aligned}
& \text { Absolute mean difference } \\
& =\frac{\times 100(\text { expressed as a percentage })}{\text { Overall mean of scores }}
\end{aligned}
$$

where the "absolute mean difference is the mean of the absolute value in the differences in the scores of the two readers, and the "overall mean of scores" is the mean of the scores of the two readers for all subjects included. A frequency distribution of HRCT score differences was also conducted for the 80 individual scores, by patient and by case (there were 5,009 cases, over two visits that were evaluable) to demonstrate the pattern of scoring for each of the two readers. Ninety-seven percent of the observations had a maximum score difference of 1 or less. Maximum difference $=($ maximum of the four scores, E1, E2, S1, $\mathrm{S} 2)$ - (minimum of the four scores E1, E2, S1, S2). Only nine of the observations had maximum score differences of 3 or more. Correlations between specific HRCT changes and total HRCT scores: Correlation statistics measure the degree of linear relationship between two variables. Pearson Product Moment Correlation Coefficient is used for continuous variables. An R-value closer to 1 indicates a strong positive correlation; a value close to -1 indicates a strong negative correlation. Values close to zero indicate there is no linear relationship between the two data sets.

\section{RESULTS}

Thirty-two patients were enrolled and randomized to one of the two treatment groups. Thirty-one patients completed the study. One patient in the placebo group dropped out of the study after few days complaining about the taste of the "medication." Patients were asked to bring empty vials at the end of the study to evaluate adherence rate. Adherence rate was calculated at $86 \%$ for the study patients.

Baseline clinical characteristics of the two groups are presented in Table 1. Most of the study population had mild to moderate lung disease as indicated by CXR brasfield score, mean $\mathrm{FEV}_{1}$, and $\mathrm{FEF}_{25 \%-75 \%}, 21.55$, $89.7 \%$, and $75.9 \%$, respectively. CXR was done only at baseline to further evaluate disease severity and as part of routine $\mathrm{CF}$ care. Patients randomized to TSI were younger and had milder lung disease than those randomized to Placebo, although these differences were not statistically significant. The difference in mean weight between the two groups at baseline was statistically significant at $P=0.04$.

Mean changes in HRCT component scores between treatment arms from visit 1 to visit 2 were analyzed for consistency with the global total HRCT score and the observed change in pulmonary function. Six of the eight HRCT component scores were positively correlated with the global total score $(r>0.66)$; however, atelectasis and inhomogeneity were not strongly correlated $(r=0.42$ and $r=0.50$ respectively) and were observed to be highly discordant with the observed changes in the global total HRCT score, the HRCT component scores, and the PFT changes. Inhomogeneity was scored on expiratory images. A modified total HRCT score was calculated by dropping these two discordant component categories from the overall total score (Table 2), resulting in a larger observed HRCT treatment effect in the TSI group. The modified total score decreased $-6.68 \pm 3.04($ mean \pm SD) for TSI subjects and increased $0.02 \pm 2.0$ (mean $\pm \mathrm{SD}$ ) for the placebo subjects $(P=0.07)$ (Table 3$)$. We calculated sample sizes that would be required to show statistical significance using a modified total HRCT score, a global total HRCT score, $\mathrm{FEF}_{25 \%-75 \%}$, or $\mathrm{FEV}_{1}$. A total of 60 ,

TABLE 1-Baseline Characteristics of Study Population

\begin{tabular}{lccc}
\hline & TOBI & Placebo & $P$-value* \\
\hline Number of patients & 16 & 16 \\
Gender (M/F) & $6 / 10$ & $6 / 10$ \\
Age (years) & $11.81 \pm 7.46^{* *}(6.0-34.7) * * *$ & $15.86 \pm 7.25(7.4-28.8)$ & 0.13 \\
Weight (kg) & $34.83 \pm 14.08(17.6-62.0)$ & $46.38 \pm 16.92(21.9-73.3)$ & 0.04 \\
Weight for age (percentile) & $25 \%$ & $10 \%$ & 0.09 \\
FEV & $95.73 \pm 17.21(55.0-134.1)$ & $83.71 \pm 21.07(45.00-108.73)$ \\
FEF predicted & $81.71 \pm 22.94(29.35-111.83)$ & $70.04 \pm 31.19(17.36-110.14)$ \\
HRCT global total score & $27.77 \pm 17.64(10.00-78.00)$ & $32.80 \pm 18.24(6.25-76.25)$ & 0.24 \\
CXR brasfield score & $22.05 \pm 1.32(18.8-24)$ & $21.05 \pm 2.88(14.8-25)$ & 0.42 \\
Sweat chloride (meg/L) & $101.1 \pm 19.84(70.0-136.6)$ & $106.8 \pm 18.03(80.0-145.0)$ & 0.22 \\
Genetic analysis & 8 & 11 & 0.58 \\
$\quad$ Homozygous delta F508 & 2 & 2 & \\
$\quad$ Heterozygous delta F508 & & & \\
\hline
\end{tabular}

*Student's $t$-test for two independent samples 95\% level of significance (two-tailed).

$* *$ Mean \pm SD.

****Range. 
TABLE 2-Changes in HRCT Individual Category Scores Between Visits 1 and 2 and the Difference in Mean Change Between TSI and Placebo Groups

\begin{tabular}{|c|c|c|c|c|c|c|c|}
\hline \multirow[b]{2}{*}{ HRCT Scores } & \multicolumn{2}{|c|}{ TSI mean score } & \multirow{2}{*}{$\begin{array}{c}\text { TSI mean } \\
\text { score change }\end{array}$} & \multicolumn{2}{|c|}{ Placebo mean score } & \multirow{2}{*}{$\begin{array}{l}\text { Placebo mean } \\
\text { score change }\end{array}$} & \multirow{2}{*}{$\begin{array}{c}\begin{array}{c}\text { Difference in mean } \\
\text { score change }\end{array} \\
\text { TSI - Placebo }\end{array}$} \\
\hline & Visit 1 & Visit 2 & & Visit 1 & Visit 2 & & \\
\hline Global total score & 27.78 & 23.72 & 4.06 & 32.80 & 32.63 & 0.17 & 4.23 \\
\hline Bronchiectasis & 4.03 & 3.48 & 0.55 & 5.41 & 5.45 & $(0.04)$ & 0.59 \\
\hline Peribronchial thickening & 7.95 & 6.71 & 1.24 & 9.08 & 9.54 & $(0.46)$ & 1.70 \\
\hline Mucous plugging & 0.30 & 0.39 & $(0.09)$ & 0.56 & 0.69 & $(0.13)$ & 0.04 \\
\hline Atelectasis/consolidation & 0.34 & 0.48 & $(0.14)$ & 0.47 & 0.13 & 0.34 & -0.48 \\
\hline Nodular opacities & 2.04 & 1.11 & 0.93 & 2.20 & 2.06 & 0.14 & 0.79 \\
\hline Linear opacities & 2.93 & 1.98 & 0.95 & 3.25 & 3.33 & $(0.08)$ & 1.03 \\
\hline Inhomogeneity & 3.63 & 6.11 & $(2.48)$ & 7.24 & 8.95 & $(1.71)$ & -0.77 \\
\hline Hyperinflation & 6.57 & 3.45 & 3.12 & 4.74 & 4.06 & 0.68 & 2.44 \\
\hline
\end{tabular}

100,200 , and over 800 patients, respectively would be required to demonstrate significance in this patient population (Table 4).

The inter-observer coefficient of variation for the global total HRCT score was $19.9 \%(P=0.000)$. Inter-observer differences in reading scores is observed in about $1 / 3$ of the total (Table 6).

The intra-observer difference in the mean HRCT reading for the first reader was $P=0.260$ and for the second reader, the $P$-value was 0.087 . Non-parametric tests (Wilcoxon exact) were performed for each reader and for both readers combined (Table 5). The results of this analysis were consistent with the $t$-test results. Table 5 showed significance only for reader 2 for atelectasis with student's $t$-test 0.027 and Wilcoxon 0.020 . However, it was noted that $97 \%$ of the observations had a maximum score difference of 1 or less (maximum score $=$ maximum of the four reading scores - minimum of the four reading scores). Interestingly, the interval between the two readings for the first reader was 1-2 weeks while the interval for the second reader was 1-2 months. A frequency distribution of HRCT score differences was also conducted for the 80 individual scores, by patients and by case over the two visits, to demonstrate the pattern of scoring for each of the two readers. Ninety-seven percent of the observations had a maximum score difference of 1 or less (maximum score $=$ maximum of the four reading scores - minimum of the four reading scores). We analyzed the quality of life data (CFQ). No useful results were obtained from the analysis due to the small number of patients in each age group.

\section{DISCUSSION}

PFTs are considered the standard for assessment of lung disease in CF. However, conventional PFTs are not very sensitive in the detection of early lung damage. ${ }^{16}$ In addition, lung function tests are an indirect measure of structural integrity of the airways. ${ }^{17}$ HRCT was shown to be a more sensitive measure of detecting structural abnormalities than CXR in a recent study. ${ }^{4}$ HRCT showed that airways undergo substantial thickening early in life in CF patients. ${ }^{4}$ HRCT has the potential to be a useful outcome measure in CF. A necessary attribute of an outcome measure is that it improves rapidly with effective therapy. ${ }^{7}$ This is a second study that was conducted by our group to evaluate the value of HRCT as an end-point in a double blind placebo controlled study. ${ }^{4}$ In this study, we demonstrated that HRCT could be a more sensitive objective measure than PFTs in detecting a treatment effect in CF pulmonary disease.

Previous studies evaluated the usefulness of HRCT in understanding the natural history of different lung disease. In asthma, a study was conducted comparing airtrapping as quantified by HRCT of the chest compared to lung function and airway inflammation in children with mild to moderate disease. ${ }^{18}$ The data suggested that the quantitative HRCT may be a useful tool in the evaluation of peripheral airflow obstruction in children with asthma. ${ }^{18}$ A second study showed that patients with chronic obstructive pulmonary disease (COPD) have more prominent HRCT findings as compared with asthmatics. ${ }^{19}$ In another study, HRCT provided a complete and precise assessment of children with bronchiectasis. ${ }^{20}$ Ventilation/ perfusion scans and lung functions were found to be additive tools to the understanding of the complexity of the disease process. ${ }^{20}$ Several studies were done in CF to evaluate the severity and progression of lung disease using HRCT. $^{2,7,13,21,22}$ Other studies have evaluated early lung disease in CF using HRCT. ${ }^{14,17,23,24}$ These studies addressed the natural history of the pulmonary disease rather than an intervention. Our study investigated HRCT as an outcome surrogate in a short-term intervention study.

Using HCRT to measure treatment outcomes in patients with mild to moderate disease could result in smaller number of patients required to demonstrate a significant 
TABLE 3-Mean Changes in FEV Predicted, FEF $_{25 \%-75 \%}$, Total HRCT Global and Modified Global Scores From Baseline to End of Study

\begin{tabular}{|c|c|c|c|c|}
\hline \multirow{2}{*}{ FEV predict } & \multicolumn{2}{|c|}{ TSI } & \multicolumn{2}{|c|}{ Placebo } \\
\hline & & & & \\
\hline Visit 1 & $95.73 \pm 17.21 *$ & $(55.0,134.1)^{* *}$ & $83.71 \pm 21.07$ & $(45.00,108.73)$ \\
\hline Visit 2 & $97.03 \pm 16.60$ & $(66.17,134.94)$ & $84.46 \pm 20.85$ & $(40.23,107.68)$ \\
\hline$P$-value & \multicolumn{4}{|c|}{0.97} \\
\hline \multicolumn{5}{|c|}{$\mathrm{FEF}_{25 \%-75 \%}$ predicted } \\
\hline Visit 1 & $81.71 \pm 22.94$ & $(29.35,111.83)$ & $0.04 \pm 31.19$ & $(17.36,110.14)$ \\
\hline Visit 2 & $87.78 \pm 24.77$ & $(31.17,122.56)$ & $68.16 \pm 30.66$ & $(18.30,99.58)$ \\
\hline$P$-value & \multicolumn{4}{|c|}{0.23} \\
\hline \multicolumn{5}{|c|}{ Global HRCT total score } \\
\hline Visit 1 & $27.78 \pm 17.64$ & $(10.0,78.0)$ & $32.80 \pm 18.23$ & $(6.25,76.25)$ \\
\hline Visit 2 & $23.72 \pm 12.02$ & $(9.0,52.5)$ & $32.63 \pm 17.65$ & $(7.25,67.75)$ \\
\hline$P$-value & \multicolumn{4}{|c|}{0.13} \\
\hline \multicolumn{5}{|c|}{ Modified HRCT total score } \\
\hline Visit 1 & $23.81 \pm 14.54$ & $(6.75,60.5)$ & $25.24 \pm 17.22$ & $(5.00,73.25)$ \\
\hline Visit 2 & $17.13 \pm 8.86$ & $(8.5,40.75)$ & $25.26 \pm 14.91$ & $(6.75,63.5)$ \\
\hline$P$-value & \multicolumn{4}{|c|}{0.07} \\
\hline
\end{tabular}

$*$ Mean $\pm \mathrm{SD}$

**Range.

treatment effect. Our calculation showed that 100 patients would be sufficient to demonstrate statistical significance, compared to 200 and over 800 patients if we used $\mathrm{FEF}_{25 \%-75 \%}$ and $\mathrm{FEV}_{1}$ respectively. When the HCRT score was modified by elimination of the two discordant scores (inhomogeneity and atelectasis) from the global total score, the number of patients needed to show statistical significance decreased to 60 patients. Enrolling smaller number of patients would allow faster completion of studies, decreased numbers of patients exposed to overall radiation, and decreased costs in conducting studies. In addition, fewer patients would be subjected to investigational medications.

Peribronchial thickening, linear opacities, nodular opacities, hyperinflation, and to a lesser degree, bronchiatasis and mucus plugging, were the most concordant with the global total score (Table 2), and observed improvement in PFTs. In a previous study, changes in peribronhial thickening or airway thickening correlated significantly with the change in $\mathrm{FEF}_{25 \%-75 \%}$ predicted, a lung function test believed to be sensitive to abnormalities in the peripheral airways. ${ }^{21}$ Previous studies have shown that bronchiatasis and mucus plugging were most sensitive components of CF lung disease. ${ }^{21,22}$ However, these studies were evaluating progressive damage and longitudinal decline in lung disease. Our study was done in mild-moderate $\mathrm{CF}$ patients; for this patient population, a modified HRCT score might be more sensitive.

A trend towards improvement was noted in the HRCT score compared to baseline in the TSI group, although not statistically significant, while no change was observed in the placebo group (Table 2). Axial HRCT images in

TABLE 4-Sample Sizes Required to See Statistically Significant Differences Between Mean Changes in TSI and Placebo Groups

\begin{tabular}{|c|c|c|c|c|c|c|}
\hline & \multirow{2}{*}{$\begin{array}{l}\text { Mean change } \\
\text { Visit } 1-\text { Visit } 2 \\
( \pm 95 \% \mathrm{CI})\end{array}$} & \multicolumn{5}{|c|}{ Sample sizes } \\
\hline & & 31 & 60 & 100 & 200 & 800 \\
\hline \multicolumn{7}{|c|}{ Modified HRCT total score } \\
\hline TSI & -6.68 & $(-13.27,-0.09)$ & $(-11.1,-2.26)$ & & & \\
\hline Placebo & .02 & $(-4.22,4.26)$ & $(-2.72,2.76)$ & & & \\
\hline \multicolumn{7}{|c|}{ Global HRCT total score } \\
\hline TSI & -4.06 & $(-10.32,2.21)$ & & $(-7.60,-0.51)$ & & \\
\hline Placebo & -.17 & $(-1.75,5.24)$ & & $(-0.17,3.66)$ & & \\
\hline \multicolumn{7}{|c|}{$\mathrm{FEF}_{25 \%-75 \% \text { predicted }}$} \\
\hline TSI & 6.08 & $(3.43,15.59)$ & & & $(2.27,0.88)$ & \\
\hline Placebo & -0.6 & $(-5.19,3.98)$ & & & $(-2.38,0.17)$ & \\
\hline \multicolumn{7}{|c|}{ FEV predicted } \\
\hline TSI & 1.29 & $(-5.25,7.83)$ & & & & $(-0.02,2.60)$ \\
\hline Placebo & 1.17 & $(-1.58,3.92)$ & & & & $(0.63,1.70)$ \\
\hline
\end{tabular}


HRCT in Evaluating TSI in Cystic Fibrosis

TABLE 5-Summary of Changes in HRCT Score From V1-V2 by Each Reader and by Both Readers Combined

\begin{tabular}{|c|c|c|c|c|c|c|}
\hline \multirow[b]{2}{*}{ Components } & \multicolumn{2}{|c|}{ Reader 1} & \multicolumn{2}{|c|}{ Reader 2} & \multicolumn{2}{|c|}{ Both readers combined } \\
\hline & Student's $t$-test & $\begin{array}{c}\text { Wilcoxon exact } \\
P \text {-value }\end{array}$ & Student's $t$-test & $\begin{array}{c}\text { Wilcoxon exact } \\
P \text {-value }\end{array}$ & Student's $t$-test & $\begin{array}{c}\text { Wilcoxon exact } \\
P \text {-value }\end{array}$ \\
\hline Bronchiectasis & 0.280 & 0.226 & 0.605 & 0.773 & 0.288 & 0.267 \\
\hline Peribronchial thickening & 0.054 & 0.079 & 0.113 & 0.219 & 0.044 & 0.121 \\
\hline Mucous plugging & 0.896 & 0.692 & 0.894 & 0.482 & 0.875 & 0.423 \\
\hline Atelectasis & 0.861 & 0.148 & 0.027 & 0.020 & 0.294 & 0.090 \\
\hline Nodular opacities & 0.575 & 0.760 & 0.353 & 0.206 & 0.394 & 0.404 \\
\hline Linear opacities & 0.154 & 0.331 & 0.078 & 0.066 & 0.058 & 0.147 \\
\hline Inhomogeneity & 0.639 & 0.428 & 0.965 & 0.661 & 0.841 & 0.452 \\
\hline Hyperinflation & 0.627 & 0.264 & 0.270 & 0.451 & 0.373 & 0.268 \\
\hline Global & 0.260 & 0.154 & 0.087 & 0.342 & 0.112 & 0.227 \\
\hline
\end{tabular}

inspiration at two levels from a patient in the TSI group at baseline and end of study are shown in Figure 1. Using the modified HRCT total score in this mostly mild-moderate CF lung disease population seems reasonable. This post hoc analysis is potentially useful in developing an improved measurement tool, although larger prospective studies are needed to further evaluate this. The coefficient of variation is considered by some to be a useful single measure of the amount of variation in a distribution relative to the magnitude of the mean of the distribution and is often expressed as a percentage/ratio. We used the formula described in Nasr et al. ${ }^{4}$ The coefficient of variation for the HRCT global score was $19.9 \%$. That was lower than that found in Nasr et al. $(49.1 \%),{ }^{4}$ but higher than the $5 \%$ reported by Santamaria, et al. ${ }^{14}$ The higher range variation in HRCT scores in the Nasr study was attributed both to the small range of the scoring system and the small number of patients $(\mathrm{n}=12){ }^{4}$ The higher interobserver coefficient of variation (19.9\%) observed in this study probably reflects the small number of patients and the range of scoring scale for HRCT. One would hope to find no differences in the mean scores either within each reader or between the two readers. The coefficient of variation is probably the most sensitive to any difference

TABLE 6-Maximum-Minimum Difference Frequency Check

The FREQ procedure

\begin{tabular}{lcccc}
\hline Difference & Frequency & Percent & $\begin{array}{c}\text { Cumulative } \\
\text { frequency }\end{array}$ & $\begin{array}{c}\text { Cumulative } \\
\text { percent }\end{array}$ \\
\hline 0 & 3,138 & 62.65 & 3,138 & 62.65 \\
1 & 1,727 & 34.48 & 4,865 & 97.13 \\
2 & 135 & 2.70 & 5,000 & 99.82 \\
3 & 6 & 0.12 & 5,006 & 99.94 \\
4 & 3 & 0.06 & 5,009 & 100.00 \\
& \multicolumn{4}{c}{ Frequency missing $=31$} \\
\hline
\end{tabular}

between the raters on individual cases. A mean is a summary measure, and can therefore mask differences at the individual level. In addition, two readers scores can be highly correlated and still vary greatly, for example, if one rater's scores are always exactly twice those of a second rater, they will have a correlation of 1.0, with the best fit line between their scores having a slope of two. Their coefficient of variation will still be high. The intraobserver variability showed lower reproducibility with longer intervals (1-2 months) between the two readings. This is consistent with other studies. ${ }^{5}$
A

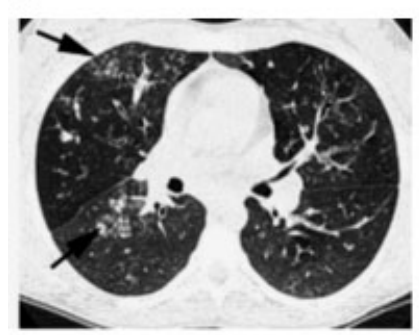

C

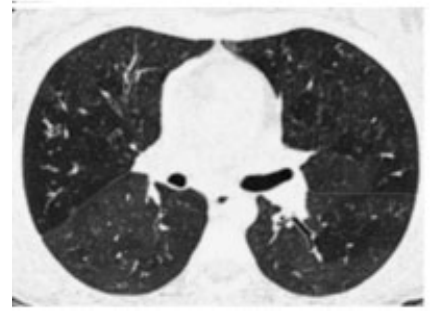

B

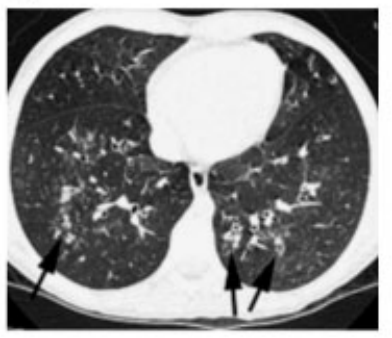

D

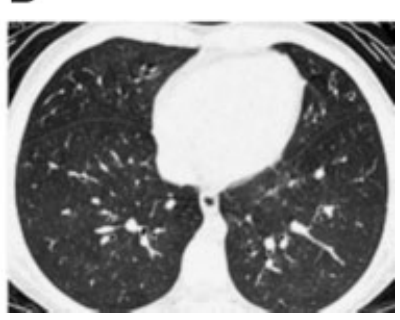

Fig. 1. A-D: Girl (13-year-old) from the TSI group. Axial HRCT images in inspiration obtained before therapy $(A, B)$ and 4 weeks after starting therapy $(C, D)$. On the initial examination (A, B), scattered nodular opacities are seen within both lungs (arrows), more on the right. Bronchial walls appear mildly thickened diffusely. On the followup examination (C, D), the nodular opacities have resolved and the bronchial walls do not appear as thick. Note slight inhomogeneity of aeration, persisting after therapy (C). 
There are several limitations of this study. The first is that it is a pilot. A larger study would be of interest to further evaluate the usefulness of this measure in $\mathrm{CF}$ patients with mild to moderate lung disease. Patients randomized to TSI were younger and had milder lung disease; however, there was no statistical significance between the two groups.

A second limitation is the use of limited cuts of the HRCT which reduces the sensitivity to detect changes. The limited number of cuts could have also contributed to the high interobserver coefficient of variation. However, using limited cuts reduces radiation dosing that patients are exposed to. In this protocol, there were specific instructions on how and where to place the five slices at baseline and end of study. This should result on each slice being in the same vicinity of the previous one, but not the exact position. We felt that the five scans during inspiration and five during expiration are reasonably representative of the disease present and changes that occurred at the end of study. The effective and equivalent doses of radiation from HRCT in this study was approximately equal to two CXRs (PA and lateral view each), even though the radiation dose is delivered in a different manner for both procedures (effective dose was 0.04 and $0.02 \mathrm{mSv}$ and the equivalent doses were 0.09 and 0.04 mGy respectively).

A third limitation is the method of evaluating expiratory images. We did not control for lung volume during scanning as done in another study. ${ }^{23}$ Although landmarks were used to identify levels, it was difficult to exactly match imaging levels from examination to examination. Findings on expiratory images (hyperinflation and inhomogeneity) were dependent on the patient following breath holding instructions closely. A suboptimal expiratory effort could mimic hyperinflation or mask inhomogeneity. The importance of monitoring volume has been reported by Robinson, et al. ${ }^{13}$ Lack of volume monitoring may have increased the variability of this potentially useful component. Further studies are needed with volume monitoring to further analyze the value of measuring inhomogeneity in this patient population.

A fourth limitation was our inability to reliably analyze the CFQ data. Patient reported outcomes and quality of life measures are increasingly being recognized as important parameters in measuring treatment effect. Larger studies are needed to evaluate $C F Q$ value as an end point in mild CF lung disease.

\section{CONCLUSION}

- HCRT can be a sensitive measure of change in mild to moderate $\mathrm{CF}$ pulmonary disease.

- HRCT is likely to require a smaller sample size than $\mathrm{FEV}_{1}$ or $\mathrm{FEF}_{25 \%-75 \%}$ to demonstrate a significant treatment effect in patients with mild to moderate lung disease.
- Atelectasis and inhomogeneity may not be useful component scores in evaluating HRCT in mild to moderate CF lung disease.

- The limited number of slices with low mA and/or scan time reduces the radiation dose.

\section{REFERENCES}

1. Burns JL, Ramsey BW, Smith AL. Clinical manifestations and treatment of pulmonary infections in cystic fibrosis. Adv Pediatr Infect Dis 1993;8:53-66.

2. Dorlöchter L, Nes H, Fluge G, Rosendahl K. High resolution CT in cystic fibrosis-The contribution of expiratory scans. EJR 2003;47:193-198.

3. Mayo JR, Jackson SA, Müller NL. High resolution CT of the chest: radiation dose. AJR 1993;160:479-481.

4. Nasr SZ, Kuhns LR, Brown RW, Hurwitz ME, Sanders GM, Strouse PJ. Use of computerized tomography and chest X-rays in evaluating efficacy of aerosolized recombinant human DNase in cystic fibrosis patients younger than age 5 years: A preliminary study. Pediatr Pulmonol 2001;31:377-382.

5. de Jong PA, Ottink MD, Robben SG, Lequin MH, Hop WCJ, Hendricks JJE, Paré PD, Tiddens HAWM. Pulmonary disease assessment in cystic fibrosis: Comparison of CT scoring systems and value of bronchial and arterial dimension measurements. Radiology 2004;231:434-439.

6. Konstan MW, Byard PJ, Hoppel CL, Davis PB. Effect of highdose ibuprofen in patients with cystic fibrosis. N Engl J Med 1995;332:848-854.

7. Brody AS, Molina PL, Klein JS, Rothman BS, Ramagopal M, Swartz DR. High-resolution computed tomography of the chest in children with cystic fibrosis: Support for use as an outcome surrogate. Pediatr Radiol 1999;29:731-735.

8. Ramsey BW, Pepe MS, Quan JM, Otto KL, Montgomery AB, Williams-Warren J, Vasiljev K-M, Borowitz D, Bowman CM, Marshall BC, Marshall S, Smith AL. Intermittent administration of inhaled tobramycin in patients with cystic fibrosis. N Engl J Med 1999;340:23-30.

9. Murphy TD, Anbar RD, Lester LA, Nasr SZ, Nickerson B, VanDevanter DR, Colin AA. Treatment with tobramycin solution for inhalation reduces hospitalizations in young $\mathrm{CF}$ subjects with mild lung disease. Pediatr Pulmonol 2004;38:314-320.

10. Quittner AL, Sweeny S., Watrous M, Munzenberger P, Bearss K, Nitza AG, Fisher LA, Henry B. Translation and linguistic validation of a disease-specific quality of life measure for cystic fibrosis. J Pediatr Psychol 2000;25:403-414.

11. Brasfield D, Hicks G, Seng-Jaw S, Tiller RE. The roentgenogram in cystic fibrosis: A new scoring system. Pediatrics 1979;63:2429 .

12. Robinson TE, Leung AN, Northway WH, Blankenberg FG, Chan FP, Bloch DA, Holmes TH, Moss RB. Composite spirometriccomputed tomography outcome measure in early cystic fibrosis lung disease. Am J Respir Crit Care Med 2003;168:588-593.

13. Robinson TE, Leung AN, Northway WH, Blankenberg FG, Bloch DA, Oehlert JW, Al-Dabbagh H, Hubli S, Moss RB. Spirometrytriggered high-resolution computed tomography and pulmonary function measurements during an acute exacerbation in patients with cystic fibrosis. J Pediatr 2001;138:553-559.

14. Santamaria F, Grillo G, Guidi G, Rotondo A, Raia V, de Ritis G, Sarnelli P, Caterino M, Greco L. Cystic fibrosis: When should high-resolution computed tomography of the chest be obtained? Pediatrics 1998;101:908-913.

15. Shah RM, Sexauer W, Ostrum BJ, Fiel SB, Friedman AC. Highresolution $\mathrm{CT}$ in the acute exacerbation of cystic fibrosis: 
Evaluation of acute findings, reversibility of those findings, and clinical correlation. AJR 1997;169:375-380.

16. Becklake MR, Permutt S. Evaluation of tests of lung function for "screening" for early detection of chronic obstructive lung disease. In: The lung in the transition between health and disease. New York, NY: Marcel Dekker, 1979.

17. Tiddens HAWM. Detecting early structural lung damage in cystic fibrosis. Pediatr Pulmonol 2002;34:228-231.

18. Jain N, Covar RA, Gleason MC, Newell JD, Gelfand EW, Spahn JD. Quantitative computed tomography detects peripheral airway disease in asthmatic children. Pediatr Pulmonol 2005;40:211218.

19. Harmanci E, Kebapci M, Metintas M, Ozkan R. High-resolution computed tomography findings are correlated with disease severity in asthma. Respiration 2002;69:420-426.

20. Pifferi M, Caramella D, Bulleri A, Baldi S, Peroni D, Pietrobelli A, Boner AL. Pediatric bronchiectasis: Correlation of HRCT, ventilation and perfusion scintigraphy, and pulmonary function testing. Pediatr Pulmonol 2004;38:298-303.

21. de Jong PA, Nakano Y, Lequin MH, Mayo JR, Woods R, Paré PD, Tiddens HA. Progressive damage on high resolution computed tomography despite stable lung function in cystic fibrosis. Eur Respir J 2004;23:93-97.

22. Brody AS, Molina PL, Klein JS, Campbell JD, Millard SP, Quan J. High-resolution CT is more sensitive to longitundinal decline in lung status in young children with $\mathrm{CF}$ than pulmonary function tests. Pediatr Pulmonol 2003;36:A388.

23. Long FR, Williams RS, Castile RG. Structural airway abnormalities in infants and young children with cystic fibrosis. J Pediatr 2004; 144:154-161.

24. Bonnel A-S, Song SM-H, Kesavarju K, Newaskar M, Paxton CJ, Bloch DA, Moss RB, Robinson TE. Quantitative air-trapping analysis in children with mild cystic fibrosis lung disease. Pediatr Pulmonol 2004:38:396-405. 